\title{
Endoscopic Transsphenoidal Management of Ecchordosis Physaliphora in the Sphenoid Sinus: A Case Report and Review of Approaches to Resection
}

$T$ Logan Lindemann, $B S^{1}$, Brandon Kamrava, $M D^{1}$, Baidarbhi Chakraborty, $M D^{2}$, Amandeep Aneja, $M D^{2}$, Scott Shepard, $M D^{3}$ and Pamela C Roehm, MD, Ph. $D^{1,3,4^{*}}$

${ }^{1}$ Departments of Otolaryngology-Head and Neck Surgery, Lewis Katz School of Medicine, Temple University, USA

${ }^{2}$ Pathology and Laboratory Medicine, Lewis Katz School of Medicine, Temple University, USA

${ }^{3}$ Neurosurgery, Lewis Katz School of Medicine, Temple University, USA

${ }^{4}$ Neuroscience, Lewis Katz School of Medicine, Temple University, USA

\begin{abstract}
Background: Ecchordosis Physaliphora (EP) is a benign lesion originating from the developing notochord, similar to chordoma. We report complete resection of an asymptomatic, extradural EP in the sphenoid sinus using an endoscopic transsphenoidal approach and review surgical approaches to resection.

Case description: A 35-year-old woman presented with headache and a family history of multiple sclerosis. Radiologic imaging revealed a $2.6 \mathrm{~cm} \mathrm{T1}$ hypointense, T2 hyperintense lesion extending from the dorsal clivus into the posterior aspect of the sphenoid sinus. An endoscopic endonasal transsphenoidal approach facilitated inspection and complete resection of the extradural lesion. Combined clinical, radiographic, surgical, and histopathologic findings confirmed the diagnosis of EP.

Conclusions: Endoscopic approaches to resection allow for excellent visualization and resection of clival EPs. Endoscopic endonasal transsphenoidal approaches are minimally invasive options for resection of EP of the anterior clivus, particularly for lesions extending into the sphenoid sinus. While total resection is desirable, there are currently no reported EP recurrences after resection.
\end{abstract}

\section{Keywords}

Chordoma, Ecchordosis physaliphora, Endoscopic resection, Sphenoid sinus

\section{Abbreviations}

CSF: Cerebrospinal Fluid; CT: Computed Tomography; EP: Ecchordosis Physaliphora; MRI: Magnetic Resonance Imaging

\section{Introduction}

Ecchordosis physaliphora (EP) and chordoma are uncommon lesions which originate from the embryonic notochord. During normal fetal development, the notochord is reabsorbed, leaving evidence of its existence in the nucleus pulposus of the intervertebral discs [1,2]. Remnants of the notochord may be found at any level of the adult vertebral column, most commonly in the sacrococcygeal and clival regions [2,3]. EPs are discovered serendipitously, classically presenting as benign, hamartomatous intradural lesions. Clival chordomas typically present symptomatically as malignant, extradural neoplasms. However, differentiation between EP and chordoma is often difficult. While most cases of EP are asymptomatic, cases of symptomatic EP have been reported [4]. Similarly, asymptomatic chordoma may be incidentally identified on imaging evaluation for other conditions. Defining genetic features for diagnosis of EP have not been identified [4]. Surgery with excisional biopsy is recommended for

* Corresponding author: Pamela C Roehm, M.D. Ph.D, Department of Otolaryngology-Head and Neck Surgery, Lewis Katz School of Medicine, Temple University, 3440 N Broad St, Kresge West, Suite 300, Philadelphia, PA, 19140, USA, Tel: 215-7073665, Fax: 215-707-7523

Accepted: June 11, 2019

Published online: June 13, 2019

Citation: Lindemann TL, Kamrava B, Chakraborty B, et al. (2019) Endoscopic Transsphenoidal Management of Ecchordosis Physaliphora in the Sphenoid Sinus: A Case Report and Review of Approaches to Resection. J Head Neck Surg 2(1):18-23 


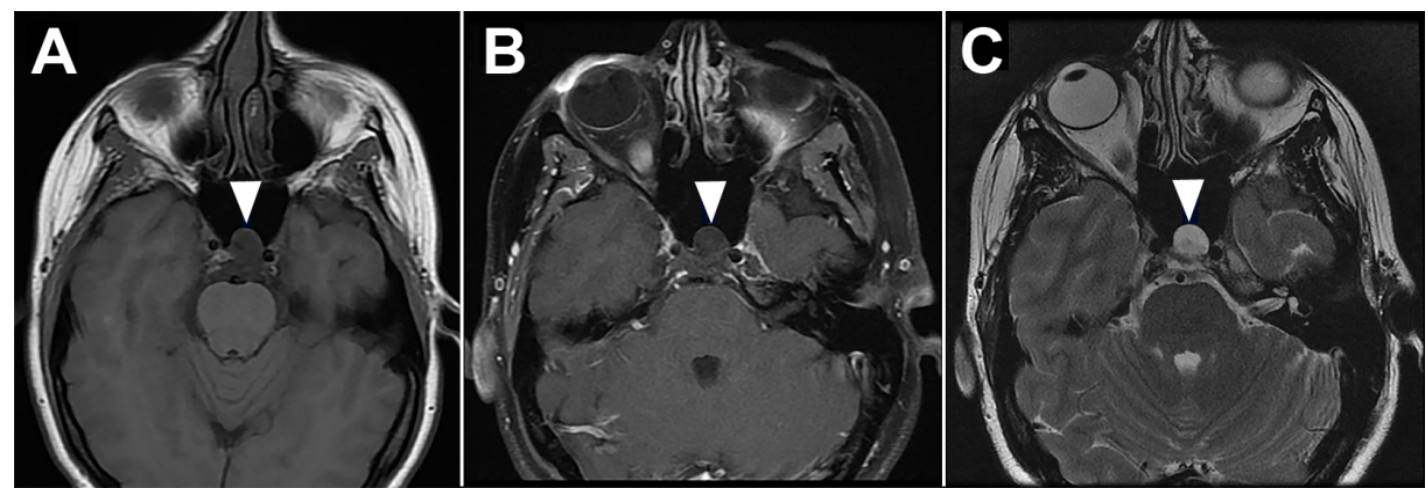

Figure 1: Magnetic resonance imaging of the patient's clival lesion, demonstrating extension into the sphenoid sinus (arrowhead). (A) T1weighted axial image taken without contrast revealing a hypointense lesion. (B) Gadolinium-enhanced T1-weighted axial image revealing no contrast enhancement. (C) T2-weighted axial image revealing a hyperintense lesion.


Figure 2: Intraoperative endoscopic images before and after ecchordosis physaliphora resection. (A) Prior to resection: the lesion in situ ( $^{*}$ ) present caudal to the sella turcica (ST), with extension into the sphenoid sinus. (B) Post-resection, endoscopic imaging demonstrates total resection of the extradural lesion.

resection of EP with definitive diagnosis made retrospectively based on a combination of pathology, radiographic features, and postoperative behavior of the lesion. Endoscopic surgical approaches can provide a minimally invasive option for diagnosis and definitive treatment of EP. Here we report a symptomatic case of extradural transclival EP treated by endoscopic endonasal transsphenoidal resection. The case is discussed in the context of the current literature and followed by a review of the surgical management of clival EP.

\section{Case Presentation}

A 35-year-old woman with a family history of multiple sclerosis presented to her primary care physician with complaints of headache. Neurological evaluation was positive for dizziness, imbalance, and headaches. The patient underwent initial magnetic resonance imaging (MRI) of the brain without contrast to rule out demyelinating disease. A T2 hyperintense clival lesion with a pedunculated component projecting into the posterior margin of the sphenoid sinus was identified. Computed tomography (CT) of her paranasal sinuses confirmed the lesion, noting extension along the posterior aspect of the clivus extending into the posterior aspect of the left sphenoid sinus. An MRI with contrast was obtained and compared with the previous CT and MRI examinations. Imaging revealed an irregular $\mathrm{T} 1$ hypointense, $\mathrm{T} 2$ hyperintense lesion that showed no appreciable enhancement with contrast (Figure $1 \mathrm{a}$, Figure $1 \mathrm{~b}$ and Figure $1 \mathrm{c}$ ). High resolution T2 imaging also demonstrated moderate heterogeneous isointense material within the posterior aspect of the lesion (Figure 1b). Total craniocaudal length of the lesion measured $2.6 \mathrm{~cm}$, while the portion extending into the left posterior sphenoid sinus measured $1.2 \times 1.2 \times 1.0 \mathrm{~cm}$. Surgical resection was recommended to rule out chordoma, given her symptoms and extension of the lesion into the sphenoid sinus.

The patient underwent endoscopic endonasal transsphenoidal resection of the lesion which was performed as a combined procedure by the neurosurgical and otolaryngology teams. Stereotactic imaging guidance was used throughout the procedure. The inferior and middle turbinates were first out fractured, and then the inferior two-thirds of each middle turbinate were removed to improve visualization of the sphenoid ostium. Right and left sphenoidotomies were performed, followed by posterior nasal septectomy to facilitate passage of instruments into the sphenoid cavity. The sella turcica and underlying transclival cyst were fully visualized (Figure 2a).The lesion was completely extradural, with no evidence of bony infiltration or remodeling. The cyst was removed in its entirety and sent for permanent pathology 
Citation: Lindemann TL, Kamrava B, Chakraborty B, et al. (2019) Endoscopic Transsphenoidal Management of Ecchordosis Physaliphora in the Sphenoid Sinus: A Case Report and Review of Approaches to Resection. J Head Neck Surg 2(1):18-23

(Figure 2b).

Using an endoscopic drill, the bone of the clivus surrounding the cyst cavity was removed. The dura of the posterior fossa was uncovered but not opened. Following removal of the tumor, the area was inspected carefully and no evidence of cerebrospinal fluid (CSF) leak was observed.

Final histopathology revealed the presence of a neurenetric cyst and tiny fragments of notochord-type tissue with physaliphorous cells embedded in a mucoid matrix (Figure 3a and Figure $3 b$ ). Immunohistochemical stains showed staining with cytokeratin AE1/E3, S100, CK18 and Brachyury (Figure $3 c$ and Figure $3 d$ ). No areas of necrosis or mitoses were observed. Clinico radiographic correlation with these findings indicated a benign notochord remnant, consistent with EP.

Nasal packing was removed after 2 days and the patient was discharged. Following excision of the mass, the patient noted significant symptom improvement, with decreased headache frequency and resolution of her vertigo. Ten months after resection brain MRI showed no evidence of recurrence.

\section{Discussion}

EP is hypothesized to form via perforation of the ascending notochord through the clivus and subsequent migration into the dura [3-5]. Several cases have also reported perfo- ration and extension into the sphenoid sinus [2,3,6-9]. The migration of notochord tissue explains the potential for physaliphorous cells to be found in the extradural, subdural, and subarachnoid spaces $[2,3,6]$.

In this case, the patient's lesion was completely extradural, attached to the clivus by a pedicle with extension into the sphenoid sinus. Although extradural EPs are unusual, it is likely that these cells did not migrate far enough to become located intradurally. Chordomas are typically extradural and infiltrate the surrounding bone, occasionally extending intradurally [10]. We observed no evidence of bony infiltration, suggesting that the clival defect was congenital and not the result of malignant infiltration.

No single diagnostic criterion has been described to differentiate between EP and chordoma. The common radiographic features of the two lesions include T1-hypointensity and T2-hyperintensityon MRI. EPs typically lack of enhancement with gadolinium, in contrast to the intense but heterogeneous enhancement seen with chordoma [11]. Absence of mitoses and a low proliferation indexon histopathology suggest EP $[12,13]$. However, the shared embryologic origin between EP and chordoma often preclude definitive differentiation. Obtaining a tissue sample for biopsy prior to resection is typically not feasible. Therefore, details of the patient's history and radiographic features are likely to be the most important factors in determining a management strategy. In the reported

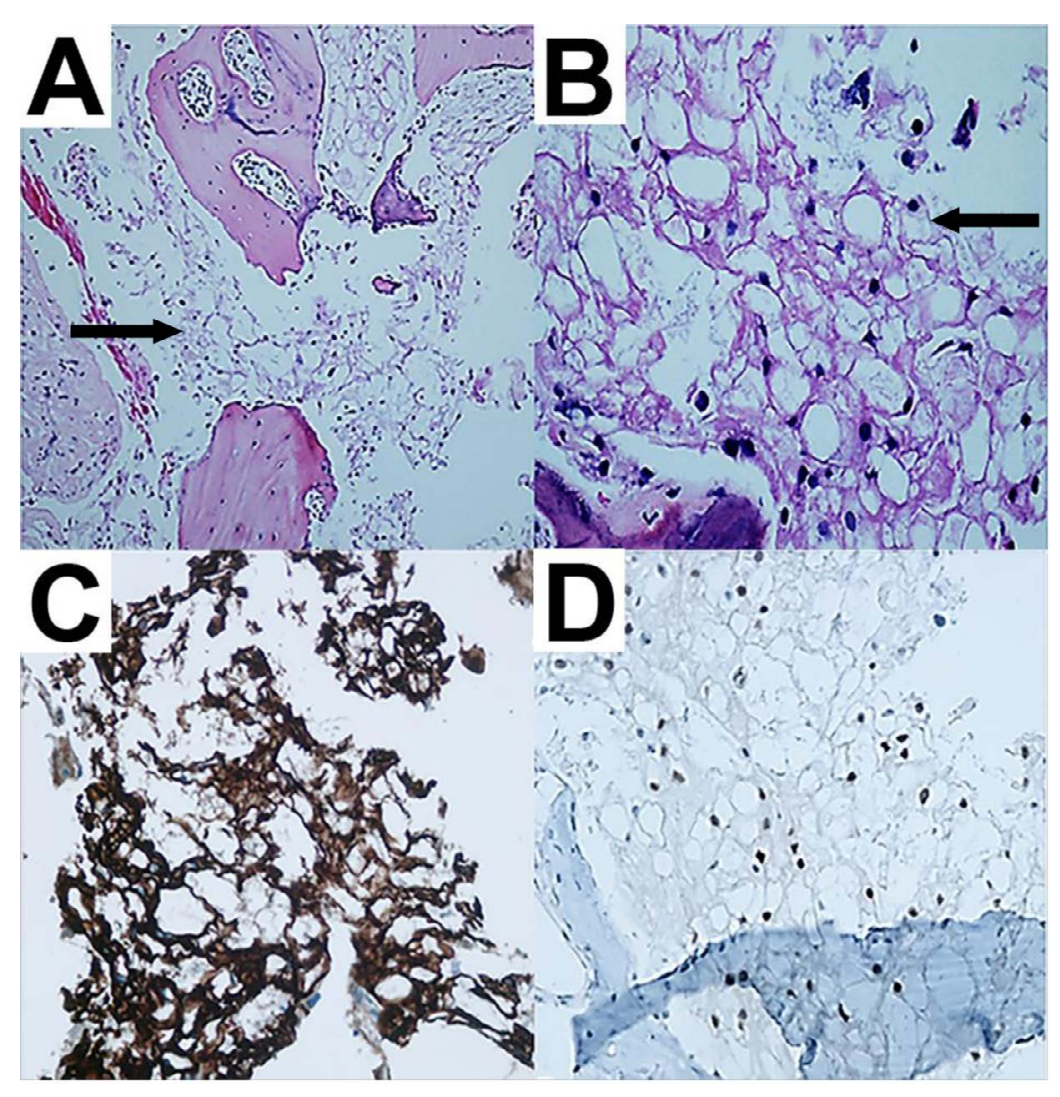

Figure 3: Histopathological examination of excised clival cyst. (A) Hemotoxylin and eosin clusters of physaliphorous cells (arrowhead) characterized by large cells with clear vacuolated cytoplasm and eccentric nuclei, 200X. (B) Magnified view of hematoxylin and eosin staining, arrowhead indicates physaliphorous cell (400X). (C) Immunohistochemistry demonstrating positive cytokeratin AE1/AE3 staining (400X). (D) Immunohistochemistry demonstrating positive Brachyury staining (400X). 
Citation: Lindemann TL, Kamrava B, Chakraborty B, et al. (2019) Endoscopic Transsphenoidal Management of Ecchordosis Physaliphora in the Sphenoid Sinus: A Case Report and Review of Approaches to Resection. J Head Neck Surg 2(1):18-23

case, there were neurologic complaints (headache) as well as the extradural location of the lesion, which increased the possibility of chordoma. Additionally, our patient was potentially predisposed to future CSF leak if the lesion grew due to its extension into the sphenoid sinus $[2,3,6,14,15]$. Thus, surgical resection was determined to be optimal management.

Traditionally, surgeons have approached the clivus and anterior skull base via craniotomy, with surgical approaches selected according to craniocaudal location. Superior clival lesions are best reached via orbitozygomatic or variant approaches, middle clival lesions via transpetrosal approaches, and inferior clival lesions via far-lateral approaches [16]. Suboccipital, presigmoidal, transpetrosal, and frontotemporal craniotomies have been employed for EP resection (Table 1).
While effective, open approaches are generally more invasive and cross more neurovascular structures than endoscopic approaches [16].

In 2002, Cha, et al. described use of an endoscope during EP resection, noting that endoscopy improved visualization of the tumor and allowed for more complete resection [12]. Endoscopy has been successfully combined with endonasal, transmaxillary, and trans-third ventricular approaches to EP resection (Table 1). Endoscopic endonasal approaches provide a direct, minimally invasive approach to anterior midline clival lesions. Other anterior approaches, such transmaxillary resection, provide similar benefits but are less functionally and cosmetically appealing. Adib, et al. describe similar midline indications for an endoscopic trans-third ventricular

Table 1: Surgically Managed Cases of Clival Ecchordosis Physaliphora.

\begin{tabular}{|c|c|c|c|c|c|}
\hline Reference & Age, Sex & Position (Size) & Approach & Resection & Outcome \\
\hline Toda, et al. [17] & $56, F$ & $\begin{array}{l}\text { Prepontine, attached to clivus } \\
(1.5 \mathrm{~cm})\end{array}$ & Lateral suboccipital & Total & NED, 2 years \\
\hline Cha, et al. [12] & $49, \mathrm{M}$ & Prepontine $(1.5 \mathrm{~cm})$ & $\begin{array}{l}\text { Transmaxillary transclival, } \\
\text { endoscopic assist }\end{array}$ & Total & NED, 18 months \\
\hline Takeyama, et al. [18] & $12, \mathrm{M}$ & Prepontine $(4.0 \mathrm{~cm})$ & N/A & Subtotal & NED, 6 months \\
\hline Rotondo, et al. [4] & $47, \mathrm{~F}$ & $\begin{array}{l}\text { Prepontine, attached to clivus } \\
\text { (N/A) }\end{array}$ & Presigmoidal & Total & NED, 1 year \\
\hline Ling, et al. [7] & $45, M$ & $\begin{array}{l}\text { Prepontine, transclival into } \\
\text { sphenoid sinus }(3.0 \mathrm{~cm})\end{array}$ & Transpetrosal & Total & NED, 4 weeks \\
\hline Alli, et al. [2] & $52, F$ & $\begin{array}{l}\text { Transclival into sphenoid } \\
\text { sinus }(1.3 \mathrm{~cm})\end{array}$ & $\begin{array}{l}\text { Endoscopic endonasal } \\
\text { transsphenoidal }\end{array}$ & $\begin{array}{l}\text { Clival defect } \\
\text { repair w/o } \\
\text { resection }\end{array}$ & CSF leak recurrence \\
\hline Miki, et al. [19] & $59, \mathrm{M}$ & Retroclival (N/A) & $\begin{array}{l}\text { Endoscopic trans-third } \\
\text { ventricular }\end{array}$ & Total & NED, 3 years \\
\hline Yamamoto, et al. [20] & $20, M$ & $\begin{array}{l}\text { Retroclival prepontine }(2.2 \\
\mathrm{cm})\end{array}$ & $\begin{array}{l}\text { Endoscopic endonasal } \\
\text { transsphenoidal }\end{array}$ & Subtotal & NED, 1 year \\
\hline Krisht, et al. [21] & $16, F$ & Retroclival $(3.0 \mathrm{~cm})$ & $\begin{array}{l}\text { Microscopic transnasal } \\
\text { transsphenoidal }\end{array}$ & Subtotal & NED, 30 months \\
\hline Kaul, et al. [8] & $52, F$ & $\begin{array}{l}\text { Transclival intosphenoid sinus } \\
(0.6 \mathrm{~cm})\end{array}$ & $\begin{array}{l}\text { Endoscopic endonasal } \\
\text { transsphenoidal }\end{array}$ & Subtotal & NED, 1 year \\
\hline Dias, et al. [6] & $54, F$ & $\begin{array}{l}\text { Transclival into sphenoid } \\
\text { sinus (N/A) }\end{array}$ & Endoscopic endonasal & Total & NED, 2 years \\
\hline $\begin{array}{l}\text { Bolzoni-Villaret, et al. } \\
{[14], \# 1}\end{array}$ & $51, F$ & $\begin{array}{l}\text { Retroclival, transclival (1.2 } \\
\mathrm{cm})\end{array}$ & $\begin{array}{l}\text { Endoscopic endonasal } \\
\text { transsphenoidal }\end{array}$ & Total & NED, 12 months \\
\hline $\begin{array}{l}\text { Bolzoni-Villaret, et al. } \\
{[14], \# 2}\end{array}$ & $39, F$ & $\begin{array}{l}\text { Retroclival, transclival into } \\
\text { sphenoid sinus }(1.5 \mathrm{~cm})\end{array}$ & $\begin{array}{l}\text { Endoscopic endonasal } \\
\text { transsphenoidal }\end{array}$ & Total & NED, 12 months \\
\hline Choudhri, et al. [5] & $63, M$ & $\begin{array}{l}\text { Prepontine, attached to clivus } \\
(2.1 \mathrm{~cm})\end{array}$ & $\begin{array}{l}\text { Endoscopic endonasal } \\
\text { transsphenoidal }\end{array}$ & Total & NED, 21 months \\
\hline Zhong, et al. [22] & $34, \mathrm{M}$ & $\begin{array}{l}\text { Prepontine, attached to clivus } \\
(3.0 \mathrm{~cm})\end{array}$ & Frontotemporal & Subtotal & NED \\
\hline Adib, et al. [23] & $57, \mathrm{M}$ & Retroclival $(1.5 \mathrm{~cm})$ & $\begin{array}{l}\text { Endoscopic trans-third } \\
\text { ventricular }\end{array}$ & Subtotal & NED, 6 months \\
\hline Filis, et al. [24] & $44, F$ & Prepontine $(1.3 \mathrm{~cm})$ & Frontotemporal & Total & NED, 1 year \\
\hline Galloway, et al. [9] & $40, F$ & $\begin{array}{l}\text { Transclival into sphenoid } \\
\text { sinus (N/A) }\end{array}$ & $\begin{array}{l}\text { Endoscopic endonasal } \\
\text { transsphenoidal }\end{array}$ & N/A & NED, 3 years \\
\hline Miki, et al. [25] & $44, F$ & Retroclival prepontine (N/A) & Anterior transpetrosal & Total & NED, 5 months \\
\hline
\end{tabular}

Abbreviations: F: Female; M: Male; N/A: Not available; NED: No evidence of disease. 
Citation: Lindemann TL, Kamrava B, Chakraborty B, et al. (2019) Endoscopic Transsphenoidal Management of Ecchordosis Physaliphora in the Sphenoid Sinus: A Case Report and Review of Approaches to Resection. J Head Neck Surg 2(1):18-23

approach. However, the approach is limited to single-instrument manipulation and is more applicable to lesions extending into the posterior clivus. Via third-ventricle approaches lesions adherent to neurovascular structures may be difficult to totally resect [23]. In our case, an endoscopic endonasal transsphenoidal approach to the clivus was used given the extension of the lesion into the sphenoid sinus.

Yamamoto, et al. reported the first successful endoscopic endonasal transsphenoidal EP resection in 2013 [20]. Eight prior cases of EP have reported successful resection using this approach. Four of these procedures achieved total resection, 2 were subtotal resections, and 2 did not specify the extent of the resection in the manuscript. Excluding one report that did not report follow-up imaging, there was no evidence of recurrence for any of these resections. Follow-up ranged from 9 months to 3 years, with a median duration of 1 year. While total resection is desirable in cases where chordoma has not yet been ruled out, no evidence of EP recurrence has yet been reported. Longer follow-up data is needed to determine the potential for EP recurrence after subtotal resection.

Previous cases report that the endoscopic endonasal transsphenoidal approach can be used to successfully manage the following complications associated with EP, including repair of clival defects $[6,8,9,14,15]$, management of CSF leaks $[6,9,14,15]$, and decompression of the abducens nerve $[14,20]$. An endoscopic endonasal approach is not always indicated for clival EP. Lesions extending lateral to the internal carotid artery should not be approached in this fashion due to risks of catastrophic bleeding if the carotid artery must be mobilized to remove tumor. Additionally, more caudally located lesions may not be fully removed through this approach alone $[5,16]$. Traditional open approaches or craniotomy combined with endoscopy may be indicated for these lesions [16].

\section{Conclusions}

Clear differentiation between EP and chordoma is often difficult. All clinical, diagnostic, and surgical features must be considered for diagnosis $[6,26]$. Minimally invasive approaches to surgery may be particularly valuable when definitive diagnosis has not yet been reached. In our case, an endoscopic endonasal transsphenoidal approach allowed for complete resection without brain retraction or dural infringement. The approach should be considered for lesions extending into the sphenoid sinus, as it provides direct access to the sinus and retroclival space.

\section{Funding}

This research did not receive any specific grant from funding agencies in the public, commercial, or not-for-profit sectors.

\section{References}

1. Stam FC, Kamphorst W (1982) Ecchordosis physaliphora as a cause of fatal pontine hemorrhage. Eur Neurol 21: 90-93.

2. Alli A, Clark M, Mansell NJ (2008) Cerebrospinal fluid rhinorrhea secondary to ecchordosis physaliphora. Skull Base 18: 395-400.

3. Macdonald RL, Cusimano MD, Deck JHN, et al. (1990)
Cerebrospinal fluid fistula secondary to ecchordosis physaliphora. Neurosurgery 26: 515-519.

4. Rotondo M, Natale M, Mirone G, et al. (2007) A rare symptomatic presentation of ecchordosis physaliphora: Neuroradiological and surgical management. J Neurol Neurosurg Psychiatry 78: 647-649.

5. Choudhri O, Feroze A, Hwang P, et al. (2014) Endoscopic resection of a giant intradural retroclival ecchordosis physaliphora: Surgical technique and literature review. World Neurosurg 82: 912.e21-912.e26.

6. Dias LA, Nakanishi M, Mangussi-Gomes J, et al. (2014) Successful endoscopic endonasal management of a transclival cerebrospinal fluid fistula secondary to ecchordosis physaliphora - An ectopic remnant of primitive notochord tissue in the clivus. Clin Neurol Neurosurg 117: 116-119.

7. Ling SS, Sader C, Robbins P, et al. (2007) A case of giant ecchordosis physaliphora: A case report and literature review. Otol Neurotol 28: 931-933.

8. KaulS, Khan O, Edem I, etal. (2013)Transclival Pseudomeningocele Secondary to Ecchordosis Physaliphora: Case Report and Literature Review. J Neurol Surg Reports 74: 92-95.

9. Galloway L, Hayhurst C (2017) Spontaneous cerebrospinal fluid rhinorrhoea with meningitis secondary to ecchordosis physaliphora. Br J Neurosurg 33: 99-100.

10. Ciarpaglini R, Pasquini E, Mazzatenta D, et al. (2009) Intradural clival chordoma and ecchordosis physaliphora: A challenging differential diagnosis: Case report. Neurosurgery 64: 387-388.

11. Lagman C, Varshneya K, Sarmiento JM, et al. (2016) Proposed Diagnostic Criteria, Classification Schema, and Review of Literature of Notochord-Derived Ecchordosis Physaliphora. Cureus 8: e547.

12. Cha ST, Jarrahy R, Yong WH, et al. (2002) A Rare Symptomatic Presentation of Ecchordosis Physaliphora and Unique Endoscope-Assisted Surgical Management. min - Minim Invasive Neurosurg 45: 36-40.

13. Mehnert F, Beschorner R, Küker W, et al. (2004) Retroclival ecchordosis physaliphora: MR imaging and review of the literature. Am J Neuroradiol 25: 1851-1855.

14. Bolzoni-Villaret A, Stefini R, Fontanella M, et al. (2014) Transnasal endoscopic resection of symptomatic ecchordosis physaliphora. Laryngoscope 124: 1325-1328.

15. Ferguson C, Clarke DB, Sinha N, et al. (2015) A Case Study of Symptomatic Retroclival Ecchordosis Physaliphora: CT and MR Imaging. Can J Neurol Sci 43: 210-212.

16. Fernandez-Miranda JC, Gardner PA, Snyderman CH, et al. (2014) Clival chordomas: A pathological, surgical, and radiotherapeutic review. Head Neck 36: 892-906.

17. Toda H, Kondo A, Iwasaki K (1998) Neuroradiological characteristics of ecchordosis physaliphora. J Neurosurg 89: 830-834.

18. Takeyama J, Hayashi T, Shirane R (2006) Notochordal remnantderived mass: Ecchordosis physaliphora or chordoma? Pathology 38: 599-600.

19. Miki T, Nakajima N, Akimoto J, et al. (2008) Neuroendoscopic trans-third ventricle approach for lesions of the ventral brainstem surface. Minim Invasive Neurosurg 51: 313-318.

20. Yamamoto T, Yano S, Hide T, et al. (2013) A case of ecchordosis physaliphora presenting with an abducens nerve palsy: A 
Citation: Lindemann TL, Kamrava B, Chakraborty B, et al. (2019) Endoscopic Transsphenoidal Management of Ecchordosis Physaliphora in the Sphenoid Sinus: A Case Report and Review of Approaches to Resection. J Head Neck Surg 2(1):18-23

rare symptomatic case managed with endoscopic endonasal transsphenoidal surgery. Surg Neurol Int 4: 13.

21. Krisht KM, Palmer CA, Osborn AG, et al. (2013) Giant ecchordosis physaliphora in an adolescent girl. J Neurosurg Pediatr 12: 328333.

22. Zhong XL, Huang B, Liu C, et al. (2015) Multiple ecchordosis physaliphora: A challenging diagnosis. Chin Med J 128: 28262828.

23. Adib SD, Bisdas S, Bornemann A, et al. (2016) Neuroendoscopic Trans-Third Ventricular Approach for Surgical Management of Ecchordosis Physaliphora. World Neurosurg 90: 701.E1-701.E6.
24. Filis A, Kalakoti P, Nanda A (2016) Symptomatic ecchordosis physaliphora mimicking as an intracranial arachnoid cyst. J Clin Neurosci 28: 171-174.

25. Miki K, Yoshimoto K, Nishimura A, et al. (2017) A Case of Ecchordosis Physaliphora in the Prepontine Cistern: A Rare Entity in the Differential Diagnosis of an Epidermoid Cyst. World Neurosurg 105: 1033.e11-1033.e14.

26. Adamek D, Malec M, Grabska N, et al. (2011) Ecchordosis physaliphora - a case report and a review of notochord-derived lesions. Neurol Neurochir Pol 45: 169-173. 